\title{
Schweizerische Konkordanz im Wandel
}

von Wolf Linder

Das schweizerische politische System ist starkem innerem Wandel unterworfen. Gemeinsamkeiten der Konkordanzregierung schwinden im Zuge wachsender parteipolitischer Polarisierung. Neue gesellschaftliche Spaltungen brechen auf. Auch ohne EUMitgliedschaft ist die Schweiz dem äußeren Druck der Globalisierung und Europäisierung ausgesetzt. Das stimuliert politische Innovation, führt aber auch zu neuen Konflikten und institutionellen Veränderungen. Im nachfolgenden Beitrag wird die These vertreten, dass konsensuale Entscheidfindung in einer übergroßen Regierungskoalition als institutioneller Zwang den politischen Wandel überdauert. Die zunehmende Abhängigkeit des Landes von globalen Entwicklungen, der Verlust alter Vorteile des schweizerischen Sonderfalls sowie die passive Anpassung an die Europäisierung werfen allerdings die Frage auf, wie weit der bilaterale Weg, der die Beziehungen zur EU prägt, für die Schweiz noch begehbar ist.

The Swiss political system is changing. Consensus democracy is confronted with growing polarisation amongst the political parties and the emergence of new societal cleavages. Though not a member of the EU, Switzerland is exposed to the pressures of globalisation and Europeanisation. This stimulates political innovation on the one hand but brings new conflict and institutional change on the other. This contribution holds that an oversized government coalition and consensual decision-making are an institutional constraint and will therefore outlast political change. Growing dependence from global developments, the loss of traditional advantages of the Swiss Sonderfall, and passive adaptation to Europeanisation raise critical questions. The policy of bilateral treaties with the EU may become inadequate for successfully positioning Switzerland in the future.

\section{Krisenzeichen}

Bis heute vermittelt das schweizerische politische System wie kaum ein anderes das Bild politischer Stabilität. Ohne Machtwechsel zwischen Regierung und Opposition bilden vier Parteien eine große Koalitionsregierung, die sich auf drei Viertel der Sitze in den Parlamentskammern stützt und in kaum veränderter parteimäßiger Zusammensetzung fast fünf Jahrzehnte überdauert hat. Föderalismus und direkte Demokratie lassen keine großen politischen Sprünge zu. Sie sorgen aber, zusammen mit der politischen Konkordanz, für einen überschaubaren, bescheidenen Staat, für den Ausgleich zwischen den Regionen und den sozialen Schichten und gewähren den verschiedenen Sprachgruppen politisch-kulturellen 
Freiraum. Die vergleichende Politikwissenschaft charakterisiert das schweizerische System als Musterbeispiel einer konsensualen Demokratie im Sinne Lijpharts $^{1}$, die eher als das Gegenmodell der Konkurrenzdemokratie in der Lage ist, die Konflikte einer kulturell segmentierten Gesellschaft durch Verhandeln und Kompromiss zu lösen und die Gefahr tieferer politischer Spaltung zu überwinden. ${ }^{2}$ Dieses Bild hat Risse bekommen. Der Aufstieg der national-konservativen Schweizerischen Volkspartei (SVP) zur stärksten Kraft und die Schwächung der bürgerlichen Mitte haben die politischen Verhältnisse im Land nachhaltig verändert. Die Polarisierung zwischen der Rechtspartei der SVP und dem Links-Grünen Lager von Sozialdemokraten und Grünen hat in Sache und politischem Stil zugenommen und zur Verhärtung der politischen Fronten geführt, was manche als Verlust der alten Kultur politischer Konkordanz betrachten. Ende 2007 wurde der de-facto-Parteiführer der SVP aus der Landesregierung abgewählt. Das führte zum Ergebnis, dass die wählerstärkste Partei im Bundesrat während eines Jahres nicht mehr vertreten war, was von vielen Kommentatoren als Krise oder gar als Ende der Konkordanz gedeutet wurde. Zunehmende Wählervolatilität und veränderte Kräfteverhältnisse sind nicht das einzige, was die schweizerische Konkordanz zu verkraften hat. Innere gesellschaftliche Spaltungen in wichtigen Fragen tun sich auf. Außenpolitischer Druck nimmt zu, wie zum Beispiel 2009 seitens der OECD sowie den Nachbarländern Deutschland und Frankreich, welche von der Schweiz dezidiert die Anpassung ihrer Steuergesetzgebung und des Bankkundengeheimnisses verlangten. Die Schweiz war es gewohnt, Vorteile des Landes als Neutraler und außerhalb der EU zu suchen und zu finden. Heute schwinden viele dieser Vorteile, während die Risiken der außenpolitischen Verletzbarkeit und Isolation, aber auch des Verlusts politischer Reputation im Ausland zunehmen.

Der nachfolgende Beitrag zeigt auf, dass diese aktuellen Krisenzeichen schweizerischer Konkordanz auf längerfristige Veränderungen in den gesellschaftlichen Konfliktlinien und dem Parteiensystem zurückgehen. Er geht sodann der Frage nach, wie weit die politische Konkordanz im Umfeld wachsender innerer Polarisierung und unter dem äußeren Druck der Globalisierung zu bestehen vermag. Schließlich wird argumentiert, dass die Schweiz, als Nicht-Mitglied der EU, ihre

1 Lijphart, A.: Patterns of Democracy. Government Forms and Performance in Thirty-Six Countries, New Haven, CT, 1999.

2 Zu Gesamtdarstellungen des schweizerischen Systems: Linder, W.: Schweizerische Demokratie. Institutionen, Prozesse, Perspektiven, 2. Aufl., Bern, 2005 sowie Klöti U. u. a.: Handbuch der Schweizer Politik, 4. Aufl., Zürich, 2006. 
Beziehungen zu Brüssel in der Vergangenheit aktiv zu entwickeln vermochte. Dagegen scheinen die künftigen Perspektiven des bilateralen Wegs keineswegs gewiss.

\section{Veränderungen des Parteiensystems}

Die Parteienlandschaft, einst von geringer Volatilität geprägt, ist in Bewegung. Die Schweizerische Volkspartei (SVP), einst die Juniorpartei in der Regierung, hat ihren Wähleranteil über drei Wahlen hinweg mehr als verdoppelt (vgl. Tabelle 1) und ist als nationalkonservative Rechtspartei zur stärksten Kraft im Land geworden. ${ }^{3}$ Die ehemals tonangebenden Mitteparteien - die Freisinnig-Demokratische Partei (FDP) und die Christlichdemokratische Volkspartei (CVP) - haben einen erheblichen Teil ihrer Wählerschaft verloren, während Sozialdemokraten (SP) und Grüne (GPS) mit wechselndem Glück ein Wählersegment bewerben, das insgesamt nicht größer wird. Mehrere Kleinparteien, unter ihnen die linke Progressive Organisation (POCH) oder die „Migros-Partei“ des Landesrings der Unabhängigen (LdU) haben nicht überlebt; die einstigen Überfremdungsparteien sind in der SVP aufgegangen, und Anfang 2009 hat sich die kleine liberale Partei Schweiz (LPS) der Freisinnigen Partei angeschlossen.

Die Dynamik dieser Entwicklung ging von der SVP aus. Ihr Aufstieg wurde lange Zeit auf einen persönlichen Erfolg ihres Parteiführers Blocher zurückgeführt. Tatsächlich hat dieser die Geschicke der Partei nachhaltig geprägt. 1992 führte er mit Vehemenz die Opposition gegen die Teilnahme der Schweiz am EWR-Vertrag mit der EU an und führte sie gegen das ganze wirtschaftlichpolitische Establishment zum Erfolg. Die volkstümlich-polternde Art Blochers kam an und prägte bald einen ungewohnt aggressiven Stil eines Großteils der Partei. Vom Pfarrerssohn zum Grossunternehmer geworden, verfügte Blocher über Ressourcen, die Haushalte der Schweiz flächendeckend mit seinen persönlichen Botschaften zu bedienen und die SVP als einzige der Parteien zu einer professionellen Organisation aufzurüsten. Blochers Parteiführung wurde außer-

3 Aktuelle Daten und Berichte über die Entwicklung der schweizerischen Politik sind über InternetPlattformen verfügbar: die jährliche Chronik schweizerischer Politik Année politique suisse (APS) samt Wahl- und Abstimmungsdaten unter http://www.anneepolitique.ch, Swissvotes, die eidgenössischen Volksabstimmungen seit 1874 mit Links zu den offiziellen Dokumenten, unter http://www. swissvotes.ch, die statistischen Auswertungen von Wahlen und Abstimmungen unter http://www.bfs. admin.ch unter der Rubrik „Politik“ sowie die Vox-Analysen, eine Auswertung von Umfragen zu den eidgenössischen Volksabstimmungen unter http://www.polittrends.ch/vox-analysen. 
Tabelle 1: Parteistärken 1991-2007

\begin{tabular}{|c|c|c|c|c|c|}
\hline \multirow{2}{*}{ Partei } & \multicolumn{3}{|c|}{$\begin{array}{l}\text { Stimmenanteile in \% } \\
\text { (Nationalratswahlen) }\end{array}$} & \multicolumn{2}{|c|}{$\begin{array}{c}\text { Sitze im } \\
\text { Nationalrat }\end{array}$} \\
\hline & 1991 & 1999 & 2007 & 1991 & 2007 \\
\hline \multicolumn{6}{|l|}{ Regierungsparteien } \\
\hline Freisinnig-Demokratische Partei (FDP) & 20,9 & 19,9 & 15,8 & 44 & 31 \\
\hline Christlichdemokratische Volkspartei (CVP) & 17,8 & 15,8 & 14,5 & 35 & 31 \\
\hline Schweizerische Volkspartei (SVP) ${ }^{\mathrm{a}}$ & 11,8 & 22,5 & 28,9 & 25 & 62 \\
\hline Sozialdemokratische Partei (SP) & 19,0 & 22,5 & 19,5 & 42 & 43 \\
\hline Summe Regierungsparteien & 69,5 & 80,7 & 78,7 & 146 & 167 \\
\hline \multicolumn{6}{|l|}{ Nicht-Regierungsparteien } \\
\hline Grüne & 6,4 & 5,0 & 9,6 & 14 & 20 \\
\hline Grün-Liberale & - & - & 1,4 & - & 3 \\
\hline Liberale & 3,0 & 2,2 & 1,9 & 10 & 4 \\
\hline Alternative Linke & 4,0 & 1,8 & 1,1 & 4 & 1 \\
\hline Übrige & 17,1 & 10,3 & 7,3 & 26 & 5 \\
\hline Summe Nicht-Regierungsparteien & 30,5 & 19,3 & 21,3 & 54 & 33 \\
\hline
\end{tabular}

Anmerkung: ${ }^{\text {a }}$ Werte inklusive der Bürgerlich-Demokratischen Partei (BDP), die sich 2008 von der SVP abspaltete.

Quelle: APS (Année politique suisse), Institut für Politikwissenschaft, Bern, 1991, 1999, 2007, http://www.anneepolitique.ch.

halb wie innerhalb als autoritär kritisiert. Der Erfolg der Partei verdankte sich zunächst übergelaufenen Wählern aus rechten Splitterparteien, die mittlerweile verschwunden sind. Der anhaltende Erfolg hat allerdings tiefere Gründe. ${ }^{4}$ Die Opposition gegen die europäische Integration, die Behauptung nationaler Unabhängigkeit und klassischer Neutralität, die Begrenzung der Zuwanderung von Flüchtlingen und Migranten, Sozialstaats- und Bürokratiekritik wurden zu dauerhaft und erfolgreich besetzten Themen. Die SVP verbindet sie mit einer Kritik an der classe politique, die insofern populistisch zu nennen ist, als die Partei selbst die Vorherrschaft in der schweizerischen Politik anstrebt. Die SVP, bis vor

4 Kriesi, H.P. u.a.: Der Aufstieg der SVP. Acht Kantone im Vergleich, Zürich, 2005. 
zwei Jahrzehnten auf die protestantisch-ländlichen Regionen der Deutschschweiz beschränkt, etablierte sich auch in katholischen Kantonen, in städtischen Regionen sowie in der Romandie. Trotz ihrer neo-liberalen Wirtschaftspolitik verstand sie es, ihre gewerbliche Basis zu behalten und zusätzlichen Anhang aus den Schichten mit tieferen Einkommen und geringerer Ausbildung an sich zu binden. Da sich diese Wählerschaft der SVP nicht allein aus ökonomisch-opportunistischen Interessen, sondern auch aus konservativ-nationalen Wertvorstellungen konstituiert, ist sie als dauerhaftes Wählerpotential einzuschätzen.

Damit zeichnen sich nachhaltige Veränderungen im Parteiensystem $a{ }^{5}{ }^{5}$ Der einst geschlossene Bürgerblock von FDP, CVP und SVP findet sich zwar noch in vielen wirtschafts- und finanzpolitischen Fragen, präsentiert sich der Wählerschaft jedoch heute als gespaltenes Lager. Die SVP als dezidierte Rechtspartei bezeichnet sich als einzige echte bürgerliche Partei und sucht die Hegemonie über die dezimierten FDP und CVP, die zur politischen Mitte geworden sind. SP und Grüne kooperieren trotz ihres Wettbewerbs um das gleiche Wählerpotential. Dies sind die gebildeten Mittelschichten, vornehmlich die Beschäftigten der öffentlichen Dienste, während sich die unteren Schichten der SVP zugewandt haben. Der längerfristige Trend verschwindender Kleinparteien wurde neuerdings gebrochen durch zwei neue Abspaltungen. Die Bürgerlich Demokratische Partei entstand im Zuge der Nichtwahl Blochers 2008 durch Repräsentanten der SVP, die von ihrer Partei ausgestoßen wurden. Die Gruppierung der Grün-Liberalen spaltete sich von den Grünen ab und spricht das bürgerliche Segment der ökologisch Motivierten an.

\section{Neue Gesellschaftspolitische Spaltungen}

Der Wandel des schweizerischen Parteiensystems geht auf ähnliche Gründe wie in anderen europäischen Demokratien zurück. Parteibindungen nehmen ab, was vor allem auf Kosten der Traditionsparteien geht, die Transformation oder das Entstehen neuer Parteien begünstigt und insgesamt zu höherer Wählervolatilität führt. Solche Erscheinungen gehen aber letztlich zurück auf veränderte gesellschaftliche cleavages oder sozial-strukturelle Spaltungen, die von den Parteien aufgenommen, thematisiert und zur Mobilisierung ihres Segments der Wähler-

5 Kriesi, H.P./Trechsel, A.: Switzerland. Continuity and Change in a Consensus Democracy, Cambridge, 2008, 84-98. Zur Entwicklung auf kantonaler Ebene: Ladner, A.: Typologien und Wandel: Die kantonalen Parteiensysteme im letzten Drittel des 20. Jahrhunderts, in: Schweizerische Zeitschrift für Politische Wissenschaft, 10/4 (2004), 1-30. 
schaft eingesetzt werden. Zwei solcher Spaltungen haben, als längerfristige Erscheinung, die schweizerische Politik nachhaltig verändert. Sie betreffen die Umweltpolitik und das Verhältnis der Schweiz zum Ausland.

Die erste der neuen Spaltungen, diejenige zwischen Ökonomie und Ökologie, entwickelte sich ähnlich wie in den meisten westlichen Industriestaaten. Umweltpolitik hat in der Schweiz eine lange Vergangenheit. Der Schutz des Waldbestandes bereits zu Beginn des 20. Jahrhunderts, das Gewässerschutzgesetz der 1960er Jahre, oder die Verhinderung einiger Kraftwerkprojekte mit empfindlichen Eingriffen in die Landschaften durch Volksinitiativen sind Beispiele früher punktueller Sensibilisierung und Politisierung quer durch die sozialen Schichten. Die Wachstumskritik des Club of Rome 1972 verlieh der Umweltpolitik wie in anderen Ländern ein neues Momentum, führte aber auch zu einer zunehmend konfliktbeladenen Auseinandersetzung um die konkurrierenden Ansprüche von Ökonomie und Ökologie in fast allen Politikbereichen. Aus den Umweltbewegungen entstanden die Parteigründungen der Grünen. Mobilisierend in der neuen Spaltung waren in den 1980er Jahren vor allem die Kernenergiefrage, später das weite Thema der Gentechnologie. Beide Konflikte gaben Anlass zu zahlreichen Volksinitiativen und Referenden, welche eine tiefe Spaltung der schweizerischen Stimmbürgerschaft offenkundig machten. Im Kernenergiekonflikt verlangte die eine Seite den Ausstieg, die andere offene Rahmenbedingungen für den weiteren Ausbau der Technologie. Kein Lager konnte sich durchsetzen; dagegen wurde 1990 eine Volksinitiative angenommen, welche ein Moratorium verlangte: Für zehn Jahre wurde jegliche Entscheidung über neue Kernkraftprojekte aus der politischen Agenda genommen. Mit den jüngsten Vorschlägen der Kraftwerkbetreiber zur Ersetzung alter durch neue Kernkraftanlagen wird der Konflikt, den Beobachter vor zwanzig Jahren als eigentlichen Glaubenskrieg apostrophiert hatten, neu aufgelegt.

Die zweite der Spaltungen betrifft das Verhältnis der Schweiz zum Ausland. Ein erster Dauerkonflikt geht auf die Ausländerpolitik zurück. Diese war während Jahrzehnten auf die Zuwanderung vornehmlich Niedrigqualifizierter ausgerichtet. Ausländische Arbeitnehmer verrichteten jene Arbeit, für welche sich wegen schlechter Löhne oder Arbeitsbedingungen keine Einheimischen fanden. Dabei stieg der Anteil der Ausländer an der schweizerischen Wohnbevölkerung auf fast $22 \%$. Dauernd unterschätzt wurden die Folgeprobleme der sozialen Integration, der Konkurrenz zwischen ausländischen und einheimischen Arbeitskräften in Zeiten wirtschaftlicher Rezession, sowie die Kosten neuer Unterschichtung im Bildungssystem und vielen anderen Bereichen. Opposition kam seit den 1960er 
Jahren von der Seite kleiner Überfremdungsparteien, welche zahlreiche Volksbegehren lancierten, damit aber wenig Erfolg erzielten und später in der SVP aufgingen. Letztere machte seit den 1990er Jahren zunehmend Druck und erreichte vor allem in der Asylpolitik eine Verschärfung der Aufnahmebedingungen, wie sie auch andere europäische Länder im Zuge wachsender Migration aus Drittweltländern praktizieren. Umgekehrt wurde der Zuzug weiterer ausländischer Arbeitskräfte durch die Freizügigkeitsabkommen der EU weiter liberalisiert. Der Grundkonflikt zwischen Öffnung und Schließung der Ausländerpolitik blieb damit ein Dauerbrenner, aber während langer Zeit auf ein einziges Thema fokussiert.

Letzteres änderte sich mit dem Aufkommen der Europafrage. In der wohl wichtigsten Volksabstimmung jüngerer Zeit, der EWR-Abstimmung von 1992, sagten eine hauchdünne Mehrheit des Volkes, aber 16 der 23 Kantone Nein zum Beitritt zu einem Vertrag mit der EU, welcher der Schweiz den umfassenden Zugang zum europäischen Markt ohne formelle Mitgliedschaft geöffnet hätte. Diese Mehrheit sah im Vertrag einen Bruch mit der Kultur einer traditionellen Schweiz und wähnte die Neutralität und die Souveränität des Landes gefährdet. ${ }^{6}$ So steht denn das Land formell abseits der europäischen Integration und versucht, seine Beziehungen zur EU durch bilaterale Verträge zu regeln. Diese betreffen nicht allein das Wettbewerbs- und Wirtschaftsrecht, sondern auch Bereiche des Verkehrs, der Forschung, der Polizei, der Asyl- und selbst der Kulturpolitik. Damit ist schon angedeutet, was die Gegner des EWR-Vertrags mit ihrer Option für den vermeintlichen Alleingang nicht verhindern konnten: Die Schweiz kann sich der Internationalisierung, Europäisierung und Liberalisierung nicht entziehen.

Sie stößt dabei aber auf einen starken Interessengegensatz. Die schweizerische Wirtschaft ist mit ihren internationalen Dienstleistungs- und Industrieunternehmen einerseits stark exportorientiert, andererseits von einer vielfältigen und eher binnenorientierten Struktur von Klein- und Mittelbetrieben geprägt. Die Wirtschaftspolitik vermochte während Jahrzehnten beide zu bedienen: Der Exportwirtschaft diente eine Außenhandelspolitik des freien Wettbewerbs, bei der sich die Schweiz in allen internationalen Organisationen äußerst aktiv zeigte. Zweige der Binnenwirtschaft dagegen schützte die Politik durch die Abschottung des schweizerischen Marktes sowie durch die Zulassung nicht-tariflicher Handelshemmnisse, welche durch Branchenkartelle organisiert wurden. Mit den Globali-

6 Zu den Werthaltungen, Einstellungen und dem Abstimmungsverhalten im Urnengang vom 06. 12. 1992 siehe VOX-Analyse No. 47, Zürich/Genf, 1993. 
sierungsschüben seit den 1990er Jahren ging die Möglichkeit einer solchen Doppelstrategie weitgehend verloren. Während der Exportsektor von den neuen Optionen internationaler Arbeitsteilung profitierte, ließen der Druck der WTO und die Wirtschaftsverflechtung mit der EU der schweizerischen Politik keine andere Wahl als die Öffnung des Binnenmarktes und die Übernahme von EURecht durch die eigene Gesetzgebung. Die Binnenwirtschaft ist daher weit über die Landwirtschaft hinaus einem starken und anhaltenden Strukturwandel ausgesetzt. Intensivierter Wettbewerb hat zwar einen Teil der Konsumentenpreise gesenkt, aber die Kaufkraft vieler Löhne nicht erhöht und die Chancen sicherer Arbeitsplätze vermindert. Mitte der 1990er Jahre wurde eine Arbeitslosenquote von 5,3\% registriert - ein tiefer Wert im internationalen Vergleich, aber ein ungewohnt hohes Ausmaß, wie es die Beschäftigten in der Schweiz noch nie gesehen hatten. Die Organisations- und Konfliktfähigkeit der Gewerkschaften und Angestelltenorganisationen nahm ab: Die meisten Arbeitnehmer vermochten, anders als die Kapitalseite, nicht von den erweiterten externen Optionen der Liberalisierung und Internationalisierung zu profitieren. Eine LängsschnittAnalyse der Volksabstimmungen zeigt, dass zwei der klassischen Spaltungen der Kapital-Arbeit- und der Stadt-Land-Konflikt - in den letzten Jahrzehnten in der Stimmbürgerschaft drastisch zugenommen haben. ${ }^{7}$ Politisch artikulierten sich diese Konflikte aber vor allem als Auseinandersetzung um eine internationale Öffnung oder Abschottung der Schweiz, weshalb die politologische Literatur denn auch von einer neuen Spaltung zwischen Öffnung und Abgrenzung der Schweiz spricht. Sie trennt nicht nur Anhänger verschiedenen Wertbezugs zur nationalen Identität, sondern auch die Gewinner und Verlierer der Globalisierung und spiegelt damit die oben aufgezeigten wirtschaftlichen Interessengegensätze. ${ }^{8}$

Die klassischen cleavages, wie sie Lipset und Rokkan ${ }^{9}$ als Phänomen aller europäischen Länder postulierten, werden damit von zwei neuen Spaltungen überlagert. Die schweizerischen politischen Parteien artikulieren diese Konfliktlinien nicht nur in Wahlen, sondern auch in Volksabstimmungen. Die Positionierung der Regierungsparteien und der Grünen im Allgemeinen ist in Tabelle 2 dargestellt.

7 Linder, W./Zürcher, R./Bolliger, C.: Gespaltene Schweiz - geeinte Schweiz. Gesellschaftliche Spaltungen und Konkordanz bei den Volksabstimmungen seit 1874, Baden, 2008, 53-59.

8 Kriesi, H.P./Trechsel, A., a. a.O., 96.

9 Lipset S.M./Rokkan S.: Party Systems and Voter Alignment: Cross National Perspectives, New York, 1967. 
Tabelle 2: Die Positionierung politischer Parteien in den alten und den neuen politischen Spaltungen

\begin{tabular}{|l|c|c|c|c|c|}
\hline Cleavage & Grüne & SP & CVP & FDP & SVP \\
\hline $\begin{array}{l}\text { Laizismus/ } \\
\text { Glaubenstreue, } \\
\text { Katholizismus/ } \\
\text { Protestantismus }\end{array}$ & - & Laizismus & $\begin{array}{c}\text { Früher Ka- } \\
\text { tholizismus, } \\
\text { heute z. T. } \\
\text { noch Glau- } \\
\text { benstreue }\end{array}$ & Laizismus & $\begin{array}{c}\text { Faizismer } \\
\text { heute } \\
\text { ambivalent }\end{array}$ \\
\hline $\begin{array}{l}\text { Deutschschweiz/ } \\
\text { Romandie } \\
\text { (Zentrum/Peripherie) }\end{array}$ & - & - & - & - & - \\
\hline Stadt/Land & Stadt & Stadt & Land & Stadt & Land \\
\hline Arbeit/Kapital & Arbeit & $\begin{array}{c}\text { Kapital, } \\
\text { gelegentlich } \\
\text { Arbeit }\end{array}$ & Kapital & Kapital \\
\hline Ökonomie/Umwelt & Umwelt & Umwelt & Ökonomie & Ökonomie & Ökonomie \\
\hline Öffnung/Abgrenzung & $\begin{array}{c}\text { Öffnung/ } \\
\text { Abgrenzung }\end{array}$ & Öffnung & $\begin{array}{c}\text { Öffnung/ } \\
\text { Abgrenzung }\end{array}$ & Öffnung & Abgrenzung \\
\hline
\end{tabular}

Quelle: Linder, W./Zürcher, R./Bolliger, C., a. a.O., 73 und eigene Nachträge.

Zwei der alten cleavages sind heute wenig virulent. Die Auseinandersetzung zwischen Kirchentreue und Laizismus, der Hauptkonflikt in den ersten Jahrzehnten des Bundesstaats von 1848, ist ausgekühlt. Beim Zentrum/PeripherieKonflikt, der Spaltung zwischen der deutschsprechenden Mehrheit und der rund einen Viertel ausmachenden sprachlichen Minderheiten, suchen die Parteien die integrative Verständigung über die Landesteile hinweg und vermeiden seine Politisierung. Stadt/Land-, sowie Kapital/Arbeit-Konflikte dagegen sind virulent, und die neuen Spaltungen liegen teils quer zu den alten. Das bringt Parteien zum Teil in ein Dilemma: die Sozialdemokratie etwa dann, wenn ihre ökologische Orientierung auf Kosten der Konsumenten geht, oder die Öffnungspolitik der bürgerlichen Mitte auf Kosten ihrer gewerblichen Basis. Hier dürfte einer der Gründe für die sinkende Orientierungskraft der politischen Parteien liegen, deren Parolen in Volksabstimmungen nur teilweise befolgt werden. Die Matrix weist aber auch darauf hin, dass erfolgreiche Konfliktlösungen im multidimensionalen Parteiensystem der Schweiz unterschiedliche Koalitionen erfordern. 


\section{Konkordanz als institutioneller Zwang}

Von einer tiefen Krise politischer Konkordanz sprachen die Medien nach der Bundesratswahl 2007, bei der die Bundesversammlung Bundesrat Blocher im Amt nicht bestätigte und stattdessen ein SVP Mitglied in die Landesregierung wählte, das seiner Partei nicht genehm war. Darauf drängte die Partei beide SVPVertreter im Bundesrat aus ihrer Fraktion und kündigte an, eine konsequente Oppositionspolitik zu betreiben. In der Tat war damit die arithmetische Regierungskonkordanz, nach der die Parteien die sieben Sitze im Bundesrat nach ihrer Wahlstärke beanspruchen und erhalten, nach fast 50 Jahren beendet. Zwar entsprachen je zwei Sitze des Freisinns und der Sozialdemokraten sowie der christdemokratische Sitz der Proportionalität. Die zwei restlichen Sitze aber waren nun nicht mehr von der SVP, sondern von der neu gegründeten Splittergruppe Bürgerlich Demokratische Partei (BDP) besetzt. Die Fundamentalopposition der SVP hielt allerdings nicht lange. Als ein Bundesrat der BDP Ende 2008 seinen Rücktritt nahm, packte die SVP die Gelegenheit am Schopf und präsentierte neben Blocher einen Kandidaten, der sowohl ihr wie der Mehrheit des Parlaments genehm war. ${ }^{10}$ Mit seiner Wahl ist die arithmetische Konkordanz wieder halbwegs hergestellt, und nach zwei Schritten in die Opposition tat die SVP mindestens einen zurück.

Die Episode veranlasst zur Frage, warum die SVP ihren Oppositionskurs nicht weiter verfolgte. Die Antwort verweist auf wesentliche Eigenheiten des schweizerischen Systems. Während andere machtteilige Systeme wie etwa die Niederlande sowohl den Umfang und die Zusammensetzung einer übergrossen Koalition auf dem Verhandlungsweg frei bestimmen können und damit auf wechselnde Regierungszusammensetzungen kommen, ist dieser Weg im schweizerischen System nicht gegeben. Dessen Verfassung verlangt, dass jede Veränderung der Bundeskompetenzen zwingend der Abstimmung durch Volk und Stände in Form einer Verfassungsrevision vorgelegt werden muss, und dass 50.000 Bürger gegen jedes Gesetz und einen Teil der völkerrechtlichen Verträge ein Referendum ergreifen und damit eine Volksabstimmung erzwingen können. Dieses beträchtliche Entscheidrisiko kann nur durch Zusammenarbeit der politischen Parteien vermindert werden. Die Volksrechte bilden also einen institutionellen Konkordanzzwang, der historisch zur politischen Machtteilung im Sinne Lijpharts geführt hat. ${ }^{11}$ Regieren mit knapper Parlamentsmehrheit ist daher einem hohen

10 Näheres in APS 2007, a. a. O, 30-33.

11 Neidhart, L.: Plebiszit und pluralitäre Demokratie, Bern, 1970. 
Risiko ausgesetzt, in Volksabstimmungen zu scheitern. Diese Erfahrung machte der Kanton Genf in den 1990er Jahren, als eine bürgerliche Mehrheit ohne die Sozialdemokraten regieren wollte. Umgekehrt ist der Austritt aus der Regierung für eine einzelne Partei wenig aussichtsreich. Mit obstruktiver Referendumspolitik kann sie die Regierungspolitik zwar zu lähmen versuchen, vergibt sich damit aber im Parlament die Möglichkeit auf eine aktive Mitgestaltung der Politik, was sich elektoral auf Dauer nicht auszahlen dürfte. Diese institutionellen Gründe erklären, warum sowohl SVP wie die übrigen Parteien ein Interesse an der Wiederherstellung der Konkordanz hatten, und sie sprechen für eine anhaltende Stabilität der Machtteilung in der Schweiz. Die Nicht-Wiederwahl amtierender Bundesräte, erstmals 2003 vorgekommen, dürfte wohl die personelle Besetzung der Regierung umstrittener machen, wohl kaum aber das Prinzip der arithmetischen Konkordanz, in der Vergangenheit oft als Zauberformel bezeichnet. Der Übergang zur Mehrheitspolitik wäre auf längere Sicht nur dann aussichtsreich, wenn ihre Mehrheit auch institutionelle Veränderungen durchzusetzen in der Lage wäre, die auf einen Abbau der Konkordanzzwänge hinauslaufen. Dazu gehören nicht nur die Volksrechte, sondern auch die Vetokräfte des Föderalismus und des Bikameralismus. ${ }^{12}$

\section{Konkordanz und politische Polarisierung}

Politische Machtteilung ist in der Schweiz durch die beschriebenen Konkordanzzwänge institutionell vorgegeben. Darin liegt auch ein gewichtiger Grund, dass die große Koalition in der Schweiz ohne gemeinsames politisches Programm auskommt. Ihr Funktionieren setzt aber dennoch die grundsätzliche Bereitschaft der beteiligten Parteien zur Kooperation, zur Hinnahmebereitschaft und Vertretung des politischen Kompromisses voraus. Die Polarisierung im Parteiensystem hat nun allerdings diese Bereitschaft ein Stück weit schwinden lassen. Nichts zeigt dies so deutlich wie die nachlassende Geschlossenheit der Regierungsparteien bei Volksabstimmungen. Kam der parlamentarische Kompromiss vor das Volk, so ging die große Koalition in den 1980er Jahren noch in vier von fünf Fällen geeint mit gleichlautender Parole in den Abstimmungskampf. ${ }^{13}$ Heute hat sich dieses Verhältnis genau umgekehrt: Nur noch in $20 \%$ aller Abstimmungen ist Einigkeit der Konkordanz in der direktdemokratischen Arena zu finden. In den übrigen Fällen schert mindestens eine der Regierungsparteien aus und be-

12 Dazu statt vieler: Germann, R.: Staatsreform. Der Übergang zur Konkurrenzdemokratie, Bern, 1994.

13 Linder, W./Zürcher, R./Bolliger, C., a.a. O., 69. 
dient sich der fallweisen Opposition. Dieses Recht billigen sich die Parteien zwar seit je zu. Der Grund ist einfach. Jeder Kompromiss erfordert von den Parteien die Bereitschaft zu Abstrichen an ihren eigenen Präferenzen. Mehr noch als in der parlamentarischen Politik kann bei Volksabstimmungen jede Partei in Situationen kommen, in denen sie den Kompromiss für ihre Wählerschaft für nicht zumutbar erachtet und deshalb Wählerverluste bei Unterstützung des Kompromisses befürchtet. Von der fallweisen Opposition hatte in der Vergangenheit vor allem die SP Gebrauch gemacht, weil sie sich einem geschlossenen Bürgerblock gegenübersah. In jüngerer Zeit ist die fallweise Opposition zu einem häufig genutzten Instrument auch der SVP geworden. In ihren bevorzugten Themen des Bilateralismus mit der EU, der Ausländer- und Flüchtlingspolitik, des (selektiven) Kampfs gegen Staatsinterventionismus und Bürokratie verweigerte sie den parlamentarischen Kompromiss und zielte mit ihrer Referendumspolitik auf zusätzliche Wählerstimmen. Der Abstimmungserfolg blieb bescheiden, nicht aber der Wahlerfolg auf Kosten insbesondere der Mitteparteien FDP und CVP. Diese sahen sich schlecht belohnt in ihrem Bemühen um den politischen Ausgleich. Der Umstand, dass die SVP in ihren Mitteln keineswegs zimperlich war, neben der Linken auch ihre bürgerlichen Koalitionspartner frontal angriff und politische Gegner mit Hohn und Spott bedachte, hat ihr den Vorwurf der politischen Gegner eingetragen, die Kultur schweizerischer Konkordanz zu zerstören.

Zunehmende Polarisierung und schwindende politische Gemeinsamkeiten der Parteien werden denn auch oft zur alt bekannten These verdichtet, Regierung und Parlament seien gefangen in einer gelähmten Konkordanz, die unfähig geworden sei zur Lösung der anstehenden Probleme. ${ }^{14}$ Tatsächlich lassen sich dafür wichtige Fälle anführen. Je nach politischer Position werden die geringe Konsequenz wirtschaftlicher Liberalisierung, die ungelösten Fragen der Umweltpolitik, die fehlende Neubestimmung der Aufgaben der Armee, die ungebremste Ausdehnung des Sozialstaats oder die Entscheidungsunfähigkeit für eine konzeptionelle Europapolitik genannt. Eine systematische Untersuchung zum Koalitionsverhal-

14 So etwa: Borner, S. u.a.: Schweiz AG. Vom Sonderfall zum Sanierungsfall?, Zürich, 1990 sowie: Borner S./Rentsch H. (Hg.): Wieviel direkte Demokratie verträgt die Schweiz?, Chur, 1997. Der Basler Ökonom Borner, in seiner Analyse ähnlich konsequent wie der Politologe Germann (a.a. O.), stellte einen Abbau der Volksrechte zur Diskussion, um die Möglichkeiten der Konkurrenz- und Mehrheitsdemokratie in der Schweiz zu verbessern. Gerade in die umgekehrte Richtung liefen die Überlegungen von Borners Fachkollegen: sie entdeckten die Vorzüge direktdemokratischer Entscheidung als Garantie eines schlanken Staats, dessen Politik sich stärker mit den Präferenzen der Bürgerschaft deckt als die repräsentative Demokratie. Dazu etwa: Kirchgässner, G. u. a.: Die direkte Demokratie. Modern, erfolgreich, entwicklungs-, und exportfähig, Basel, 1999. 
ten der Volkskammer des Parlaments vermittelt allerdings ein anderes Bild. ${ }^{15}$ Nullentscheide haben - wenigstens statistisch - nach wie vor Seltenheitswert, und Konkordanz ist auf parlamentarischer Ebene trotz zunehmender Polarisierung funktionsfähig geblieben. Die Analyse von rund 8.000 Entscheidungen aus den Jahren 1995-2004 erhellt die Hintergründe und zeigt zunächst die Durchsetzungskraft der einzelnen Fraktionen. Den höchsten Erfolg mit ihren Anträgen verzeichnen - trotz ihrer numerischen Schwächung - die beiden Mitteparteien FDP und CVP, während sich die polarisierenden Parteien SP und SVP im Effekt neutralisieren. Die erwähnte kantige Oppositionspolitik der SVP hat den Preis, dass Wahlerfolge nicht durch höheren Erfolg im Parlament umgesetzt werden können. Das Ergebnis, dass die Mitteparteien nach wie vor die Musik im Parlament bestimmen, kontrastiert scharf mit dem Medienbild, in dem die SVP in der Rolle des agenda setter schweizerischer Politik schlechthin erscheint. Die starke Durchsetzungskraft der bürgerlichen Mitte ist allerdings theoretisch zu erwarten, wenn man die bestehenden Fraktionsgrößen in den beiden Kammern in Betracht zieht (vgl. Tabelle 1). Sicherer Erfolg kann nach wie vor nur durch Koalitionen von mindestens drei Regierungsparteien zustande kommen. Hier ist nun bedeutsam, dass ideologische Versteifung nicht zur Blockierung der Konkordanz geführt hat. Nach wie vor finden sich je nach Politikbereich und Sachlage wechselnde Koalitionen. Diese werden von den Mitteparteien vor allem durch die CVP herbeigeführt, die weit häufiger als die FDP auch mit dem links-grünen Lager zusammenspannt.

Die Fähigkeit zu wechselnden Koalitionen in verschiedenen Politikbereichen erklärt nicht nur die starke Position der Mitte-Parteien, sondern ist von theoretischer Relevanz: Dauernde Konkordanz unter gleichen Partnern macht Sinn nur unter der Bedingung wechselnder Koalitionen, in denen die Ungewissheit über den Ausgang von Entscheidungen die Kultur der Kooperationsbereitschaft, die gegenseitige Achtung des politischen Gegners, sowie die Fähigkeit des kreativen Kompromisses ohne konstante Verlierer zu erhalten vermag. ${ }^{16}$ Darin liegt auch eine deliberative Stärke der Schweizer Politik begründet: Wie Bächtiger und Hangartner im internationalen Vergleich von Parlamentsdebatten aufzeigen, zeichnen sich letztere in der Schweiz durch höheren Respekt und mehr argumen-

15 Schwarz, D./Linder, W.: Fraktionsgeschlossenheit im schweizerischen Nationalrat 1996-2005. Studie im Auftrag der Parlamentsdienste der schweizerischen Bundesversammlung, Bern, 2007.

16 Linder. W.: Schweizerische Demokratie, a. a. O., 313-317. 
tative Überzeugungsbereitschaft aus. ${ }^{17}$ Dieses Ergebnis ist nicht auf allgemeinkulturelle Unterschiede zurückzuführen, sondern auf die spezifischen Eigenheiten der schweizerischen Machtteilung. Anders als in den Annahmen von Tsebelis' Vetospieler-Theorie ${ }^{18}$ sind die Vetopositionen der Kooperationspartner gerade nicht absolut, weil die Einstimmigkeit nur unter drei der vier Parteien erforderlich ist. Rationales Verhalten einer Partei unter dem Konkordanzzwang der Volksrechte besteht daher im Abwägen zwischen Kooperation als Regel (um die Verhandlungsmacht im parlamentarischen Kompromiss nicht zu verlieren) auf der einen, und der fallweisen Opposition als Ausnahme (um eine Wählerschaft mit stark abweichenden Präferenzen nicht zu verlieren) auf der anderen Seite. Was dagegen institutionell nicht garantiert werden kann, ist das theoretisch wichtige Zustandekommen wechselnder Koalitionen. ${ }^{19}$ Dessen Bedingungen hängen von (sach-)politischen Konstellationen ab. Mit dem Bruch im ehemals geschlossenen bürgerlichen Lager haben sich die Chancen wechselnder Koalitionen - und damit das Funktionieren der Konkordanz - seit den 1980er Jahren eher erhöht. Das ist nicht zuletzt deswegen bedeutsam, weil mit der Parlamentsreform von 1991 die bisherigen ad-hoc-Kommissionen durch ständige Fachkommissionen in beiden Kammern abgelöst wurden, was die politische Gestaltungskraft der Legislative messbar erhöhte. ${ }^{20}$

Ein internationaler Systemvergleich von Vatter $^{21}$ zeigt immerhin, dass die Schweiz 2007 seit Lehmbruchs und Lijpharts ${ }^{22}$ ersten Studien institutionell ein Stück weit weniger konkordant geworden ist. Dies bedeutet aber noch keine Annäherung an den alternativen Typus der Konkurrenzdemokratie, sondern, so Vatter, weit eher an den internationalen Normalfall der Konsensusdemokratie. Dass sie in der Schweiz trotz zunehmender Polarisierung und ohne gemeinsames

17 Bächtiger, A./Hangartner D.: When Deliberative Theory Meets Empirical Political Science: Theoretical and Methodological Challenges, in: Political Studies (2010), i. E.

18 Tsebelis, G.: Veto Players. How Political Institutions Work, Princeton, 2002.

19 McGann, A.: The Logic of Democracy: Reconciling Equality, Deliberation and Minority Protection, Ann Arbor, 2006.

20 Lüthi, R.: Die Legislativkommissionen der Schweizerischen Bundesversammlung. Institutionelle Veränderungen und das Verhalten von Parlamentsmitgliedern, Bern, 1997 sowie Linder, W.: Schweizerische Demokratie, a.a. O., 209.

21 Vatter, A.: Vom Extremtyp zum Normalfall? Die schweizerische Konsensusdemokratie im Wandel: Eine Re-Analyse von Lijpharts Studie für die Schweiz von 1997-2007, in: Swiss Political Science Review, 14/1 (2008), 1-48.

22 Lehmbruch, G.: Proporzdemokratie: Politisches System und politischer Kultur in der Schweiz und in Österreich, Tübingen, 1967; Lijphart, A.: Democracies. Patterns of Majoritarian and Consensus Government in Twenty-One Counties, New Haven, CT, 1984. 
politisches Programm der regierenden Parteien zu funktionieren vermag, ist auf zwei Hauptfaktoren zurückzuführen. Erstens besteht aufgrund der Volksrechte der bereits erwähnte institutionell vorgegebene, innere Zwang zur Kooperation in einer übergroßen Koalition. Zweitens ist die schweizerische Politik dem äußeren Druck der Globalisierung und Europäisierung ausgesetzt, auf den ich im Schlussteil zurückkomme.

Freilich sind zwei wichtige institutionelle Schwachstellen nicht zu übersehen. Die erste betrifft das System der Kollegialregierung, in welcher die sieben Bundesräte gleichberechtigt und ohne Weisungsbefugnis des jährlich wechselnden Bundespräsidenten die Regierungsgeschäfte führen. Die Überlastung dieses Gremiums und seine begrenzte Führungsfähigkeiten sind seit Jahrzehnten ein Thema. ${ }^{23}$ Hinzu kommen in jüngerer Zeit zunehmende Schwierigkeiten der Regierung, innere Konflikte so auszutragen, dass nicht jeder von ihnen durch zudringliche Medien an die Öffentlichkeit getragen oder auch gezerrt wird und damit den Eindruck eines zerstrittenen oder führungslosen Gremiums erweckt. Alle Versuche einer Regierungsreform - von der einfachen Erhöhung der Zahl der Bundesräte bis zu einem Zwei-Ebenen-Modell von Bundesrat und weisungsabhängigen Ministern - sind bis heute nicht zuletzt am Widerstand der Landesregierung selbst gescheitert. Eine zweite, neuere Schwachstelle betrifft Entwicklungen der direkten Demokratie. Die Volksinitiative erlaubt einer Gruppierung, mit 100.000 Unterschriften praktisch jedes politisches Begehren von der Förderung von Wanderwegen bis zur Abschaffung der Armee als Verfassungszusatz zur Abstimmung durch Volk und Stände zu bringen. In den letzten Jahren sind vermehrt Initiativen aufgebracht worden, die als emotionale Reaktion auf einzelne Tagesereignisse reagieren. So wurde 2004 beispielsweise auf aufgrund eines die Öffentlichkeit aufwühlenden Verbrechens erfolgreich eine Revision des Strafrechts verlangt, die kaum verfassungskonform ist. ${ }^{24}$ Auch andere Begehren jüngerer Zeit verletzen vermehrt Verfassungsrecht oder kollidieren mit dem expansiven internationalen Recht. Bundesrat und Parlament waren in der juristischen Prüfung solcher Initiativen in der Vergangenheit liberal und vertrauten auf ihre Ablehnung im Volk. Sie konnten sich darauf verlassen, dass Initiativen

23 Brühl-Moser, D.: Die schweizerische Staatsleitung im Spannungsfeld von nationaler Konsensfindung, Europäisierung und Informatisierung, Basel, 2007 sowie: Biaggini, G. u. a. (Hg.): Demokratie - Regierungsreform - Verfassungsfortbildung, Basel, 2009.

24 Die Initiative verlangte die Anordnung der lebenslangen Verwahrung von gefährlichen und nicht therapierbaren Sexual- oder Gewaltstraftätern und wurde 2004 von einer Mehrheit des Volkes und der Stände angenommen. Ihre verfassungs- und menschenrechtskonforme Umsetzung bereitet in verschiedenen Punkten kaum lösbare Schwierigkeiten. APS, a. a. O., 2000: 28, 2003: 26 f., 2004: 23 f. 
generell nur eine Erfolgschance von etwa $10 \%$ haben, und als Außenseiterinitiativen ohne Parteiunterstützung noch weniger. Anders wird es, wenn Regierungsparteien der Versuchung erliegen, populistische oder rechtsverletzende Initiativen politisch zu unterstützen. Direkte Demokratie hat die großen gesellschaftlichen Interessengegensätze im 20. Jahrhundert gut verarbeitet und oft auch einen Beitrag zu ihrer Überwindung geleistet. Sie hat dabei auch politische Polarisierung ertragen. $\mathrm{Ob}$ sie indessen eine populistische Instrumentalisierungen durch große Parteien eben so gut bewältigt, bliebt noch zu sehen. ${ }^{25}$ Die Ausweitung der Rechtskontrolle mit der Konsequenz der vermehrten Ungültigerklärung von Volksinitiativen ist nämlich angesichts der Popularität der Volksrechte ein zweischneidiges Schwert. Dies kann ähnlich amibivalente Wirkungen wie ein Parteienverbot auslösen. Was in der Form verboten wird, ist materiell nicht vom Tisch und kann als politische Forderung zusätzliche Popularität erhalten. Schließlich hat die Beliebtheit der Volksrechte auch zu deren Ausweitung geführt. Dies gilt auch für die Außenpolitik, in der früher nur die wichtigsten Entscheidungen wie beispielsweise der Beitritt zu den UN referendumspflichtig waren. Nach der Reform von 2003 nun unterstehen alle internationalen Verträge mit bedeutendem rechtsetzendem Inhalt dem fakultativen Referendum. Hier wurden neue Risiken geschaffen. Die Ablehnung einer Vorlage im Landesrecht ist unproblematisch, weil sie jederzeit durch einen späteren Vorschlag des Parlaments neu aufgelegt werden kann. Nicht so im internationalen Vertragsrecht. Ein ausgehandelter Vertrag nutzt ein window of opportunity, das sich schließen kann, und beinhaltet ein Abkommen mit einem Drittpartner, der möglicherweise nicht interessiert ist, nach der Ablehnung des Vertrags in neue Verhandlungen einzutreten. ${ }^{26}$

\section{Policy Making im Kräftefeld der Globalisierung}

Die Schweiz ist der Globalisierung zunächst auf gleiche Weise ausgesetzt wie andere Industrieländer. Mit der Öffnung der nationalen Volkswirtschaften wird nationaler Protektionismus zurückgedrängt und ein Teil des klassischen wirtschaftspolitischen Instrumentariums unwirksam. Generell wird die Reichweite autonom nationaler Politik in dem Maße zurückgedrängt, wie internationale Regelungen auch bei der nationalen Gesetzgebung zu berücksichtigen sind. Mit der Auflösung einer klaren Trennung zwischen Innen- und Außenpolitik verschwindet auch die klare Zurechenbarkeit politischer Verantwortlichkeit. Das hat

25 Linder, W./Zürcher R./Bolliger C., a.a. O., 206-214.

26 Linder, W.: Schweizerische Demokratie, a. a. O., 394-396. 
auch Konsequenzen für das institutionelle Gleichgewicht zwischen Parlament und Regierung: Letztere gewinnt an Einfluss auf die Gestaltung der Politik. Von all diesen Erscheinungen ist der schweizerische Staat auch als EU-Nichtmitglied, aber als Vertragspartner des internationalen Rechts in grundsätzlich ähnlicher Weise betroffen wie andere Länder Europas. Wie aber reagiert das Konkordanzsystem in seinem policy shaping und policy making auf die Dynamik der Globalisierung? Die Frage scheint deshalb interessant, weil konkordanten Systemen einerseits eine geringere Innovationsfähigkeit als den Konkurrenzsystemen nachgesagt wird, andererseits aber die Globalisierung in vielen Bereichen eine Beschleunigung politischen Wandels induziert.

Zunächst eine Bemerkung zur Ausgangshypothese. Die scheinbar geringe Innovationskraft der Konkordanz ist zwar das tägliche Klagelied schweizerischer Politiker, je nach politischem Standort bei unterschiedlichen Gelegenheiten. Eine europäisch-vergleichende Studie zur Wirtschafts- und Sozialpolitik zeigt allerdings, dass konkordante Systeme wie die Schweiz nicht unbedingt schlechter abschneiden als Konkurrenzdemokratien. ${ }^{27}$ Hingegen bedingen die zahlreichen Vetokräfte schweizerischer Konkordanzdemokratie - Vielparteien-, Zweikammer- und Föderalsystem, Minderheitenschutz und direkte Demokratie - den inkrementalen Weg innenpolitischer Innovation. Letztere muss über viele kleine Schritte erreicht werden, welche nur geringe Veränderungen des status quo zulassen, aber in die gleiche Richtung weisen. Typische Beispiele sind die Totalrevision der Verfassung von 1999, die als bloße Nachführung apostrophiert wurde, nachdem 1976 Anläufe einer großen Verfassungsreform gescheitert waren. Man begnügte sich daher mit der systematischen Bereinigung der über 200 Modifikationen seit 1874. Zusammen mit kleineren materiellen Änderungen war die Revision aber doch mehr als ein formales window dressing: Das Ganze wurde mehr als die Summe seiner Teile. In ähnlicher Weise gelang 2004 eine Föderalismusreform, welche den Finanz- und Lastenausgleich, die Aufgabenteilung, sowie die Beziehungen zu den Kantonen und deren Kooperation neu regelt. ${ }^{28}$ Die Reform beanspruchte mehr als ein Jahrzehnt. Ein Teil des Gelingens mag wiederum darin liegen, dass einzelne neue Ansätze, zu denen bereits positive Erfahrungen

27 Keman, H.: Politischer Konsens und Konzertierung: Innenpolitik und die Festlegung politischer Grundsätze in Westeuropa, in: Linder W./Lanfranchi P./Weibel E. (Hg.): Schweizer Eigenart - eigenartige Schweiz, Bern, 1996, 85-97.

28 Als Vergleich zwischen der deutschen und der schweizerischen Föderalismusreform s. Linder, W.: Die Deutsche Föderalismusreform - von außen betrachtet. Ein Vergleich von Systemproblemen des deutschen und des schweizerischen Föderalismus, in: Politische Vierteljahresschrift, 48/1 (2007), 3-16. 
bestanden, ein Stück weiter getrieben und unter Nutzung von Synergien zu einem Gesamtpaket zusammengefasst wurden, das eine Mehrheit der Kantone, des Parlaments und des Volkes hinter sich brachte. Einen inkrementalen, letztlich aber starken Wandel erlebte auch die Sozialpolitik. Die schweizerische Sozialparterschaft war lange geprägt nicht nur von der friedlichen Konfliktlösung zwischen Arbeitgebern und Gewerkschaften, sondern zudem vom Prinzip der Subsidiarität: Arbeitsbedingungen und Sozialleistungen sollten wenn immer möglich auf vertragliche Weise geregelt werden, und staatliche Sozialpolitik sollte nur übernehmen, was durch die Sozialpartner nicht geregelt oder geleistet werden konnte. Dieses Muster eines subsidiären, liberalen und damit bescheidenen Wohlfahrtsstaats hat sich stark verändert. Die schweizerische Sozialpartnerschaft blieb von der internationalen Liberalisierung nicht unberührt. Gewerkschaften sahen sich ab 1990 mit einer Dezentralisierung und Individualisierung der Lohnpolitik konfrontiert und kämpften mit Mitgliederschwund. Erstaunlicherweise entwickelte sich seither der liberale zu einem post-liberalen Wohlfahrtsstaat mit einem starken Ausbau der staatlichen Sozialleistungen. Er kam nach Trampusch dadurch zustande, dass die Gewerkschaften, schwächer geworden in der vertraglichen Durchsetzung, ihre Forderungen nicht mehr über die Sozialpartnerschaft, sondern als Vorschlag gesetzlicher Obligatorien und Leistungen für die gesamte Arbeitnehmerschaft im Parlament einbrachten. ${ }^{29}$ Sie hatten damit auch in Volksabstimmungen Erfolg und erreichten einen allmählichen Ausbau staatlicher So-

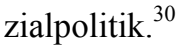

Für die globalisierte Wirtschaftspolitik jedoch konstatiert Mach eine weitgehende Ablösung des Musters inkrementaler Innovation. ${ }^{31}$ Vieles spricht für diese These. Das quantitative Wachstum der internationalen Gesetzgebung ist weit höher als dasjenige des Landesrechts. An Umfang hat das internationale Recht das Landesrecht überholt. ${ }^{32}$ Der schnellere Rhythmus gesetzgeberischer Innovation gilt vor allem in den Beziehungen zur EU, mit der zwischen 1993 und 2007 unter anderem zwei große Vertragspakete vereinbart wurden, die praktisch für

29 Trampusch, C.: Vom liberalen zu einem post-liberalen Wohlfahrtsstaat, in: Swiss Political Science Review, 14/1 (2008), 49-84.

30 Obinger, H.: Der schweizerische Sozialstaat in den 90er Jahren: Sozialpolitik unter institutionellen Bedingungen der Direktdemokratie, in: Swiss Political Science Review, 10/1 (2000), 43-63.

31 Mach, A. u. a.: Economic Regulatory Reforms in Switzerland: Adjustment Without European Integration, or How Rigidities Become Flexible, in: Journal of European Public Policy, 10/2, (2003), 302-319.

32 Linder, W./Hümbelin O./Sutter M.: Entwicklung der Rechtssetzungstätigkeit beim Bund 1983-2007eine quantitative Analyse. Studie im Auftrag der Parlamentsdienste der schweizerischen Bundesversammlung, Bern, 2009. 
die gesamte Wirtschaftspolitik bedeutsam sind. Dies mag erstaunen, weil der Verfahrensweg für beide Seiten risikoreich ist: Für die EU durch den bilateralen Weg, der die Genehmigung der Verträge durch den Rat oder die Mitgliedstaaten erfordert, und für die Schweiz, weil die Abkommen auch die Risiken eines Referendums zu bestehen hatten. Mit ihren Vereinbarungen ist die Schweiz an die Dynamik der EU-Rechtsentwicklung gebunden; sie muss diese im Vertragsrecht laufend nachvollziehen. Der Einfluss des EU-Rechts macht sich freilich über die bilateralen Verträge hinaus im gesamten Wirtschaftsrecht bemerkbar. ${ }^{33}$ Jede relevante Gesetzreform wird nach dem Kriterium ihrer Europatauglichkeit geprüft. Aufgrund der intensiven Beziehungen zu den EU-Ländern passt die Schweiz ihre Gesetzgebung laufend an Brüssel an, dies ohne Gegenseitigkeit wie jüngstens im Fall der Übernahme des Cassis-de-Dijon-Prinzips, und häufig auch über das Wirtschaftsrecht hinaus, wie bei der Bologna-Bildungsreform. Die Schweizer Behörden bezeichnen dies als autonomen Nachvollzug. Kritiker dagegen, die sich sowohl am Tempo wie an der unkritischen Übernahme aufhalten, haben dies als Ausdruck des vorauseilenden Gehorsams gegenüber Brüssel apostrophiert.

Die Frage stellt sich freilich, warum es zu solch schnellen und umfassenden Anpassungen kommt. Ein Teil der Antwort liegt auf der Hand. Die Zeit wirtschaftspolitischen Sonderfalls der Schweiz ist vorbei, weil sie sich diesen ohne gewichtige Nachteile nicht mehr leisten kann. Aber dies ist nur die Hälfte der Antwort. Ebenso bedeutsam ist, dass die Berner Politik sich diesen Sonderfall nicht mehr leisten will. Druck von außen dient vor allem den Interessengruppen der Liberalisierung dazu, ihre Anliegen verstärkt durchzusetzen. Diese Instrumentalisierung des internationalen Globalisierungsdrucks postuliert Notwendigkeiten, wo eigentlich politische Freiräume bestehen, und sie führt zur Schwächung des Einflusses der Interessengruppen der Binnenwirtschaft, vor allem jenen der ehemals starken Landwirtschaft, des Gewerbes und der Gewerkschaften.

Institutionell ist zunächst die in vielen Ländern zu beobachtende Tendenz der Gewichtsverschiebung von der Legislative auf die Exekutive zu beobachten. Im Bereich des internationalen Rechts wird das Parlament Genehmigungsinstanz,

33 Dazu etwa: Mach, A.: La Suisse entre internationalisation et changements politiques internes: législation sur les cartels et relations industrielles dans les années 1990, Zürich, 2006 oder Sciarini, P./Nicolet S.: Internationalization and Domestic Politics: Evidence from the Swiss Case, in: Kriesi, H.P. u.a. (Hg.): Contemporary Switzerland: Revisiting the Special Case, London, 2005:221-238. 
selbst dann, wenn es vorgängig vermehrt in die Außenpolitik der Regierung einbezogen wird. Auch das vorparlamentarische Verhandlungssystem verliert an Bedeutung. Schließlich kann in Einzelfällen die direkte Demokratie betroffen sein, wie das folgende Beispiel illustriert. Zugelassene Lastwagendimensionen und Gewichte waren während Jahrzehnten geringer als in den umliegenden Ländern, nicht zuletzt wegen der besonderen Verhältnisse im Alpenraum. Ihre Erhöhung war umstritten. Noch Anfang der 1990er Jahre wurde dagegen ein Referendum ergriffen. Um die Einführung größerer Abmessungen, die von der EU verstärkt verlangt wurde, zu erleichtern, verabschiedete das Parlament eine Reform des Straßenverkehrsgesetzes, das den Bundesrat ermächtigt, solche Anpassungen aus eigener Kompetenz und damit künftig ohne Referendumsrisiko vorzunehmen, wovon die Regierung denn auch sofort Gebrauch machte. ${ }^{34}$

Zusammenfassend bewirkt die Globalisierung also einen schnelleren Rhythmus gesetzgeberischer Innovation unter veränderten politischen Kräfteverhältnissen. Dabei wirkt nicht nur Druck von außen. Vielmehr wird dieser Druck von jenen Kräften genutzt, die an den Liberalisierungs- und Europäisierungsanpassungen ein direktes Interesse haben. Die verengten Handlungsspielräume nationalautonomer Politik sind also teilweise hausgemacht. Innerhalb des institutionellen Rahmens werden Veränderungen sichtbar: Wo Globalisierungsprozesse stattfinden, sinkt der Einfluss des Parlaments und werden traditionell starke Vetopunkte der Kantone, der direkten Demokratie und des vorparlamentarischen Verfahrens geschwächt.

\section{Zusammenfassung und Ausblick}

In diesem Beitrag wurde zu zeigen versucht, dass vordergründige Krisenzeichen der Konkordanz zurückgehen auf einen längerfristigen Wandel. Gesellschaftliche Modernisierung, die wie in allen Industrieländern von den wirtschaftlichen Kräften der Internationalisierung und Liberalisierung vorangetrieben wird, hat in der schweizerischen Stimmbürgerschaft die alten gesellschaftlichen Gegensätze zwischen Stadt und Land und zwischen Kapital und Arbeit verstärkt. Hinzu kommen die neuen Konfliktlinien zwischen Ökologie und Ökonomie sowie um ein offenes oder abgrenzendes Verhältnis der Schweiz zum Ausland. Ein steigendes Konfliktpotential hat zu Veränderungen des Parteiensystems mit einem partiellen Auseinanderfallen des Bürgerblocks geführt. In der schweizerischen 
Politik, stärker polarisiert als früher, sind heute drei Lager auszumachen: die zur stärksten Kraft gewordene nationale Rechte auf der einen, die stagnierenden Links-Grünen auf der andern, und eine geschwächte bürgerliche Mitte in der Rolle als Vermittler. Dabei sind die politischen Gemeinsamkeiten der vier Regierungsparteien von SVP, FDP, CVP und SP weitgehend verschwunden. Wenn politische Konkordanz, beruhend auf der übergroßen Koalitionsregierung seit bald 50 Jahren, sich dennoch gehalten hat, so liegt das aus politologischer Sicht vor allem am institutionellen Zwang der Volksrechte. Das hohe Entscheidungsrisiko direkter Demokratie zwingt die Parteien nach wie vor zur Zusammenarbeit und zum Kompromiss auf Regierungs- wie auf Parlamentsebene. Den starken Wahlerfolgen der nationalkonservativen Rechtspartei SVP steht kein entsprechend höherer Erfolg im Parlament gegenüber. Hier gibt die bürgerliche Mitte von FDP und CVP nach wie vor den Ton an. Konkordanz ist insofern sogar funktionsfähiger geworden, als sich mit der partiellen Spaltung des Bürgerblocks die Bedingungen für wechselnde Mehrheiten in den einzelnen Politikbereichen verbessert haben. Grundsätzliche Oppositionspolitik einer einzigen Partei auf parlamentarischer Ebene oder durch konsequente Referendumspolitik ist wenig aussichtsreich. Unter diesen Bedingungen ist eine Abkehr von der consensus democracy, die in der Schweiz auch durch den starken Föderalismus, den Bikameralismus und die politische Kultur des Minderheitenschutzes geprägt ist, auf absehbare Zeit wenig wahrscheinlich.

Im außenpolitischen Bereich vermag schweizerische Konkordanz den schneller und größer gewordenen Innovationsansprüchen der Globalisierung vordergründig zu genügen. Globalisierung ist allerdings mit der partiellen Entwertung von Vetopositionen in der Konkordanz verbunden: die Vertretung der wirtschaftlichen Interessen des Binnenmarkts vor allem der Landwirtschaft und des Gewerbes sowie der Gewerkschaften wird geschwächt. Umgekehrt wird der Einfluss der Regierung mit der Internationalisierung gestärkt. Konsensuale Entscheidungswege werden in Einzelfällen abgekürzt oder umgangen. Die vornehmlich reaktive Anpassung vor allem an das europäische Recht wird von den Gewinnern und Verlierern der Internationalisierung überaus unterschiedlich bewertet. Die Zunahme der Polarisierung zwischen den Parteien, das Anwachsen auch der alten Interessengegensätze zwischen Kapital und Arbeit sowie Stadt und Land sind Anzeichen dafür, dass sich mit der partiellen Entwertung von Vetopositionen der Konkordanz die künftigen Chancen einvernehmlicher Konfliktregulierung vermindern. 
Die eigentliche Herausforderung dürfte in der längerfristigen Orientierung der schweizerischen Europapolitik liegen. Der Bilateralismus, den schweizerische Politiker gerne als Königsweg bezeichnen, könnte sich, so Freiburghaus, aus verschiedenen Gründen als Sackgasse entpuppen. ${ }^{35}$ Das Verfahren, bei dem alle EU-Mitglieder oder der Rat jedem neu ausgearbeiteten Vertragswerk zuzustimmen haben, muss nicht notwendigerweise an seiner Komplexität scheitern, solange beide Seiten Interesse an der Weiterentwicklung ihrer Beziehungen haben. Die Strategie der Schweiz, die Zusammenarbeit auf ihre eigenen Interessensgebiete beschränken zu wollen, bezeichnet Freiburghaus jedoch als sektorale Illusion. Die EU kann Anpassungen auch einseitig durchsetzen, letztlich den gesamten aquis communautaire.

Teurer Bilateralismus ohne Mitwirkung in Brüssel müsste daher die EU-Mitgliedschaft als rationale Alternative erscheinen lassen. Dass eine solche im Volk und in den Kantonen eine Mehrheit finden würde, ist auf absehbare Zeit wenig wahrscheinlich. Der Mehrheit der Schweizer Bürger sind Zentralismus, die Macht der Bürokratie, die Selbstherrlichkeit politischer Eliten und die Einmischung ins Weltgeschehen suspekt. Sie sieht solche Erscheinungen nicht zuletzt verkörpert in der EU-Politik - ob zu Recht oder zu Unrecht. Die Schweiz hat mit konsensualer und direkter Demokratie, ihrem Föderalismus und der Neutralität gute geschichtliche Erfahrungen gemacht. Sie hat mit diesen Institutionen nationalen Eigennutz erzielt, aber mehr noch: sich Eigensinn gegeben. Zu kaufen ist Eigensinn - im doppelten Wortsinn persönlicher wie nationaler Identität - gerade nicht. Politische Eliten in der Schweiz stehen aber vor einer zweifachen Aufgabe: dass Eigensinn den Blick auf neue außenpolitische Realitäten nicht verstellt, und dass er sich nicht auf die reaktive Anpassung beschränkt, sondern auf eine prospektive und aktive Positionierung des Landes auszurichten vermag.

35 Freiburghaus D.: Königsweg oder Sackgasse? Sechzig Jahre schweizerische Europapolitik, Zürich, 2009. 